\title{
Development and Application of Electron Holographic Tomography for the Three- Dimensional Mapping of Electrostatic Potentials
}

\author{
Daniel Wolf and Hannes Lichte \\ Triebenberg Laboratory, Institute of Structure Physics, Technische Universität Dresden, \\ D-01062 Dresden, Germany
}

Off-axis electron holography (EH) in the transmission electron microscope (TEM) has been successfully utilized for various applications to map intrinsic electrostatic potentials and magnetostatic fields at the nanometer scale $[1,2]$. However, inherent to 2D-micrographs recorded with any imaging mode in the TEM, a phase image reconstructed by EH suffers from the drawback that the local information about the specimen along the projected dimension is lost. Thus, the mapping of electric or magnetic potentials with EH is only possible, if additional information (or assumptions) about object thickness and homogeneity can be provided.

Electron holographic tomography (EHT), i.e. EH combined with electron tomography (ET) provides a solution for this "projection problem". EHT allows quantitative 3D mapping of electrostatic potentials with a lateral resolution of a few (1-10) nanometers [3,4]. It consists of three main steps: Acquisition of a hologram tilt series, holographic reconstruction of the phase image tilt series, and tomographic reconstruction of the 3D potential. Due to the fact that the phase image is equal to the projected object potential times a well-known interaction, a tilt series of phase images represents a very suitable dataset for tomographic reconstruction: it meets the projection requirement needed for tomography.

Fig. 1 illustrates the widely automated EHT workflow that is for example achieved by using the THOMAS software package dedicated for holographic tilt series acquisition [5]. This enables revealing $3 \mathrm{D}$ potentials within a relatively small amount of time (1-2 days). For tomographic reconstruction, we use a self-developed algorithm, the Weighted Simultaneous Iterative Reconstruction Technique (WSIRT). W-SIRT tomograms provide higher resolution and fidelity than those obtained by conventional reconstruction techniques [6].

The reconstructed potentials of the nanostructures reveal their morphology and their intrinsic structure including the chemical composition. This is depicted in Fig. 2a on the example of a AlGaAs/GaAs coreshell nanowire. Moreover, functional potentials, such as the built-in voltage across p-n junctions important for the functionality of semiconductors devices (e.g. transistors), can also be analyzed (Fig. $2 b)$. This is unique and beyond the capabilities of scanning (S)TEM tomography.

\section{References:}

[1] MR McCartney and DJ Smith, Annual Reviews, 37 (2007), p. 729

[2] H Lichte and M Lehmann, Reports on Progress in Physics 71 (2008), p. 016102.

[3] PA Midgley and RE Dunin-Borkowski, Nature Materials 8, (2009), p. 271.

[4] D Wolf, H Lichte, G Pozzi, P Prete and N Lovergine, Applied Physics Letters 98 (2011), p. 264103.

[5] D Wolf, A Lubk, H Lichte and H Friedrich, Ultramicroscopy 110 (2010), p. 390.

[6] D Wolf, A Lubk and H Lichte, Ultramicroscopy submitted (2013).

[7] The authors acknowledge A. Lenk for preparing the Si sample and his help on the EHT experiments as well as all members of the Triebenberg group for their support. The research leading to these results 
has received funding from the European Union Seventh Framework Programme under Grant Agreement 312483 - ESTEEM2 (Integrated Infrastructure Initiative - I3). DW acknowledges support of the National Center for Electron Microscopy, Lawrence Berkeley Lab, which is supported by the U.S. Department of Energy under Contract \#DE-AC02-05CH11231.

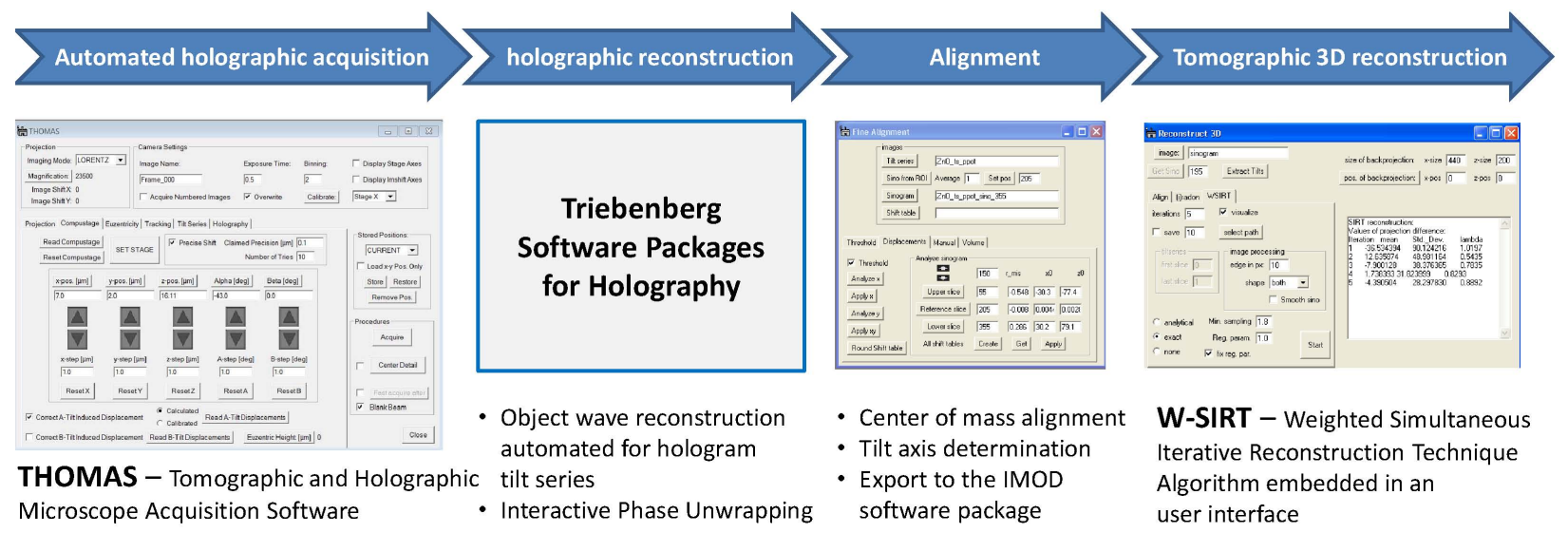

Figure 1. The laborious workflow of EHT is implemented for Gatan Inc.'s Digital Micrograph image acquisition and processing software.

(a) 3D composition

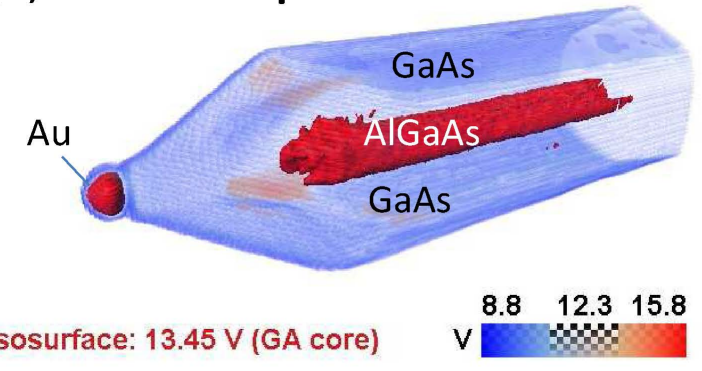

3D morphology
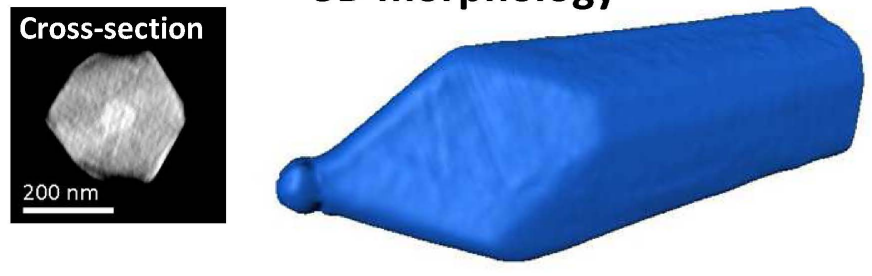

isosurface: $8.0 \mathrm{~V}$ (AGA shell)

\section{(b) 3D built-in potentials}
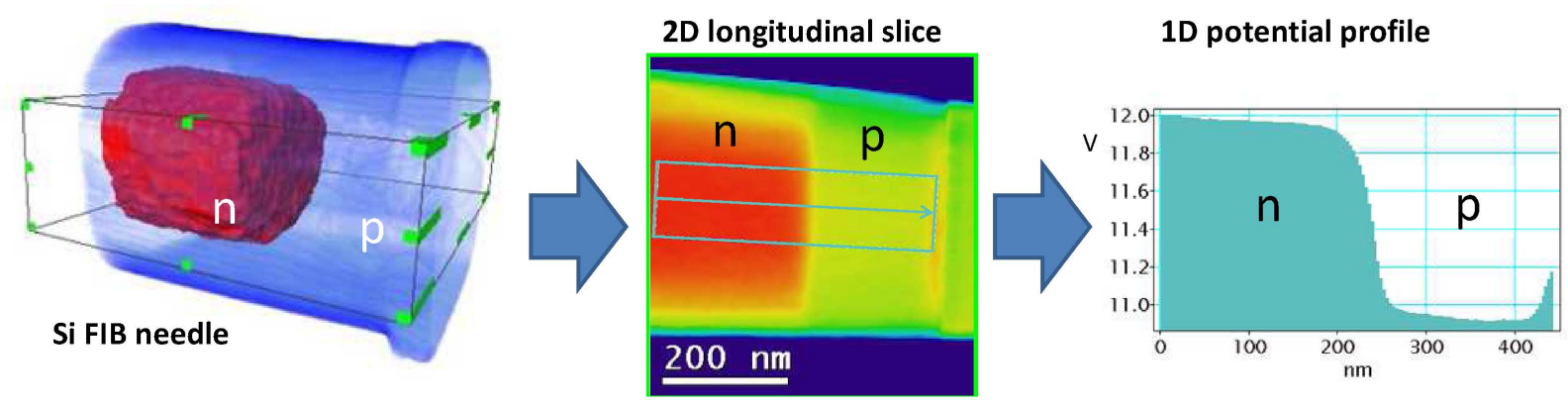

Figure 2. Application of EHT for the 3D reconstruction of nanostructures. (a) The reconstructed 3D potential of a GaAs/AlGaAs core-shell nanowire $(\mathrm{NW})$ reveals the $3 \mathrm{D}$ composition and the $3 \mathrm{D}$ morphology of the NW. The sample was kindly provided by N. Lovergine, Universita del Salento, Lecce. The experiments were performed at the FEI Titan 80-300 Berlin Holography Special in cooperation with T. Niermann and M. Lehmann from Technical University Berlin. (b) The built-in potential across a p-n junction within a silicon sample prepared by focused ion beam (FIB) can be measured inside the specimen underneath the dead layer. 